\section{Literature Cited}

1) Akehata, Takashi: Ph. D. Dissertation, Tokyo Inst. of Tech. (1957)

2) Gaffney, B. J. and T. W. Drew: Ind. Eng. Chem., 42, $1120(1950)$

3) Karabelas, A. J., T. H. Wegner and T. J. Hanratty: Chem. Eng. Sci., 26, 1581 (1971)

4) Kawazoe, K., Y. Takeuchi and T. Hashimoto:
Kagaku Kōgaku, 32, 175 (1968)

5) Kitaura, Y. and H. Tanaka: ibid., 28, 740 (1964)

6) Kitaura, Y., H. Tanaka, K. Harada and K. Araki: ibid., 29, 146 (1965)

7) Miyauchi, Terukatsu: J. Chem. Eng. Japan. 4, 238 (1971)

8) Miyauchi, T. and T. Kikuchi: ibid., 4, 336 (1971)

9) Miyauchi, T. and T. Nomura, Kagaku Kōgaku, 35, $1234(1971)$

10) Rowe, P. N.: Intern. J. Heat \& Mass Transfer, 6, 989 (1963)

\title{
SIMILARITY CONDITION OF MASS TRANSFER IN LIQUID-LIQUID SYSTEMS*
}

\author{
KORETSUNE UEYAMA AND JUN-ICHI HATANAKA \\ Department of Chemical Engineering, University of Osaka Prefecture, \\ Sakai, Japan
}

\begin{abstract}
In liquid-liquid systems, the similarity condition of the concentration boundary-layer equation of solute is obtained, assuming the tangential velocity to be linear, and the Sherwood number is expressed in an integral form. From results of its numerical integration, approximate expressions of the integral are suggested.
\end{abstract}

\section{Introduction}

When the similarity transformation can be applied to the flow of laminar boundary-layer, the mathematical treatment of fluid mechanics is much simplified because the system of partial differential equations are reduced to one involving ordinary differential equations. It is well known that such similar solutions exist when the velocity of the main stream is proportional to a power of the coordinate measured from the stagnation point ${ }^{3)}$.

In mass transfer problems in liquid phases, the boundary layer approach is often used, even in cases when the Reynolds number is not large. Because the Schmidt number is usually large in liquid phases, the non-uniform distribution of solute concentration will be restricted only to the region in the vicinity of the interface, and the existence of a concentration boundary-layer may be justified.

The condition that the boundary-layer equation governing the mass transfer rate has a similar solution is not necessarily equivalent to the condition that the velocity profile is similar. For instance, the dissolution rate from the inner surface of a circular tube can be obtained as a similar solution when linear velocity approximation near the wall is applied, but the flow is, of course, not similar.

The purpose of the present note is to derive the similarity condition of the boundary-layer equation of the solute and to provide the equation of mass trans-

* Received on March 25, 1972 fer coefficient for a given velocity profile.

It is assumed that the velocity component along the interface, $v_{x}$, of a given profile can be approximated as

$$
v_{x}(x, y)=v_{s}(x)+a(x) y
$$

Application of this linear approximation may be justified when the phase considered is liquid and $v_{s} \neq 0$.

When the Schmidt number is very large or the viscosity of the adjacent phase is very small, the velocity component in the boundary layer can be assumed to be $v_{s}(x)$. The generalized penetration theory is derived from the limiting approximation ${ }^{2,4)}$.

\section{Similarity Condition of the Governing Equation}

Axisymmetric or two-dimensional systems are shown in Fig. 1. The boundary-layer equation of solute concentration is written as

$$
\begin{gathered}
v_{x} \frac{\partial \Pi}{\partial x}+v_{y} \frac{\partial \Pi}{\partial y}=D \frac{\partial^{2} \Pi}{\partial y^{2}} \\
\Pi(0, y)=1, \Pi(x, 0)=0, \quad \Pi(x, \infty)=1
\end{gathered}
$$

The continuity equation is expressed as

$$
\frac{\partial\left(r v_{x}\right)}{\partial x}+\frac{\partial\left(r v_{y}\right)}{\partial y}=0
$$

Combining Eqs.(1) and (4), the normal velocity can be expressed as

$$
r v_{y}=-\left(r v_{s}\right)^{\prime} y-(r a)^{\prime} y^{2} / 2
$$

where the prime denotes differentiation with respect to $x$. The dimensionless concentration $I I$ is assumed to be a function only of the similarity variable $\eta$, 


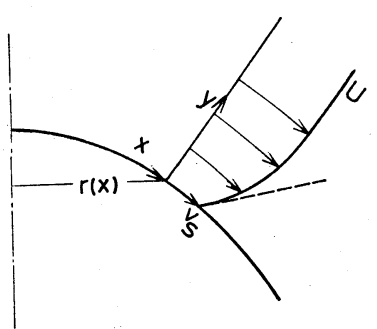

(a)

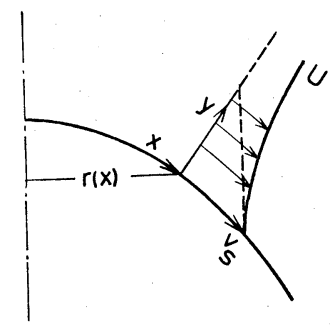

(b)
Fig. 1 Coordinates of axisymmetric system and tangential
velocity profiles

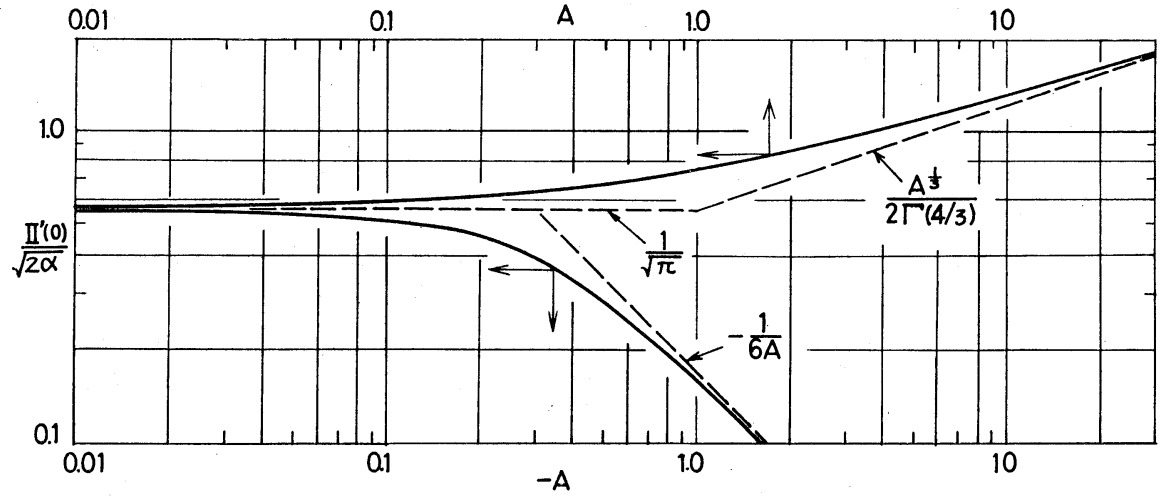

Fig. 2 Dimensionless concentration gradient at the interface

which is defined as

$$
\eta \equiv y / \delta(x)
$$

where $\delta(x)$ is an arbitrary function to be determined. Using Eqs.(5) and (6), Eq.(2) is rewritten as

$$
\begin{aligned}
& \Pi^{\prime \prime}(\eta)+\left(\alpha \eta+\beta \eta^{2}\right) \Pi^{\prime}(\eta)=0 \\
& \Pi(0)=0, \quad \Pi(\infty)=1
\end{aligned}
$$

where the prime on $\Pi$ denotes differentiation with respect to $\eta$, and $\alpha$ and $\beta$ are defined as

$$
\begin{aligned}
& \alpha D \equiv v_{s} \delta \frac{d \delta}{d x}+\frac{\delta^{2}}{r} \cdot \frac{d\left(r v_{s}\right)}{d x} \\
& \beta D \equiv a \delta^{2} \frac{d \delta}{d x}+\frac{\delta^{3}}{2 r} \cdot \frac{d(r a)}{d x}
\end{aligned}
$$

Because $\alpha$ and $\beta$ are functions only of $x$, these must be constant so as $\Pi$ to be a function only of $\eta$. The former can be arbitrarily chosen, because $\delta(x)$ is an undetermined function of $x$. Thus, we can use Eq. (10) as definition of $\delta(x)$. Under the assumption that $r v_{s} \delta=0$ at $x=0$, Eq.(10) is integrated as follows*:

$$
\begin{gathered}
\delta=\frac{\sqrt{2 \alpha D}}{r v_{s}}\left(\int_{0}^{x} r^{2} v_{s} d x\right)^{1 / 2}=\sqrt{\frac{2 \alpha \cdot D L}{v_{s}}} \\
L \equiv \frac{1}{r^{2} v_{s}} \int_{0}^{x} r^{2} v_{s} d x
\end{gathered}
$$

Then, $\beta$ is derived from Eq.(11) using Eq.(12). If $\beta$ is taken to be a constant, we can derive the following relation from Eq.(11), as shown in Appendix:

$$
B \equiv a\left(D L / v_{s}^{3}\right)^{1 / 2}=4 \beta /(2 \alpha)^{3 / 2}
$$

Inversely, if $B$ in Eq. (14) is constant, $\beta$ defined by Eq. (11) is also constant. Thus, it is shown that the similarity condition is that the dimensionless group

* In stagnation flow, $v_{s} \propto x, \delta(0)$ is not zero, but $r v_{s} \delta=0$ at $x=0$. in Eq. (14) is a constant. When both $a$ and $v_{s}$ are constant, there is no similar solution of Eq.(7), which is one of the problems discussed by Beek and Bakker ${ }^{1)}$.

\section{Calculation of Mass Transfer Coefficient}

When both $\alpha$ and $\beta$ in Eq.(7) are constant, the concentration gradient at the interface can be readily obtained as the integral form as

$$
\Pi^{\prime}(0) \cdot \sqrt{\frac{2}{\alpha}}=\left\{\int_{0}^{\infty} \exp \left(-\tau^{2}-\frac{2}{3} B \tau^{3}\right) d \tau\right\}^{-1}
$$

Local mass transfer coefficient defined by diffusional mass flux can be written as follows:

$$
k=D \Pi^{\prime}(0) / \delta(x)
$$

Then the local Sherwood number based upon the characteristic dimension $L$ can be expressed as

$$
\frac{k L}{D}=\sqrt{\frac{v_{s} L}{4 D}}\left\{\int_{0}^{\infty} \exp \left(-\tau^{2}-\frac{2}{3} B \tau^{3}\right) d \tau\right\}^{-1}
$$

The integral in Eq.(17) is divergent when the value of $B$ is negative. This may occur when the phase considered is driven by the motion of the adjacent phase. The linear approximation of the tangential velocity should be corrected as in Fig.1-(b), where the velocity component is taken to be zero in the range $y \geqq y_{0}(x)$, which is defined as follows (reference to Appendix):

$$
v_{x}(x)+a(x) \cdot y_{0}(x)=0
$$

Then, Eq.(15) becomes

$$
\begin{aligned}
\Pi^{\prime}(0) \sqrt{\frac{2}{\alpha}}= & \left\{\int_{0}^{-1 / 2 B} \exp \left(-\tau^{2}-\frac{2}{3} B \tau^{3}\right) d \tau\right. \\
& \left.-2 B \exp \left(-\frac{1}{6 B^{2}}\right)\right\}^{-1}
\end{aligned}
$$

The integrals are shown in Fig. 2, and may be approxi- 
mately expressed as follows, as results of numerical integration.

$$
\begin{aligned}
\Pi^{\prime}(0) \sqrt{2 \alpha} & =1 / \sqrt{\pi}+0.193 A^{2 / 3} \quad(0 \leqq A \leqq 1) \\
& =A^{1 / 3} /[2 \Gamma(4 / 3)]+0.189 A^{-1 / 3} \quad(A>1) \\
& =1 / \sqrt{\pi}+0.533 A \quad(-0.3 \leqq A \leqq 0) \\
& =-\frac{1}{6 A}+\frac{0.0041}{A^{3}} \quad(A<-0.3) \\
A & \equiv 2 B / 3
\end{aligned}
$$

\section{Conclusion}

The condition that the boundary-layer equation of concentration has the similar solution is that $B$ defined by Eq.(14) is constant. This parameter is a generalized form of one introduced by Beek and Bakker.

The mass transfer coefficient is expressed as a function of the Peclet number and $B$, based on the characteristic length $L$ and the interfacial velocity.

\section{Appendix}

1. Derivation of Eq.(14)

Rearranging Eq.(11), we obtain

$$
\left(\operatorname{ar} \delta^{2}\right)^{\prime}=2 \beta r D / \delta
$$

Under the condition that $\operatorname{ar} \delta^{2}=0$ at $x=0$, Eq. $(\mathrm{A}-1)$ is integrated and rewritten using Eq.(12), as

$$
\frac{2 \alpha a r D}{r^{2} v_{s}^{2}} \int_{0}^{x} r^{2} v_{s} d x=\frac{2 \beta D}{\sqrt{2 \alpha D}} \int_{0}^{x} r^{2} v_{s}\left(\int_{0}^{x} r^{2} v_{s} d x\right)^{-1 / 2} d x
$$

Substituting $L$ into Eq.(A-2), Eq.(14) is obtained.

2. Derivation of Eq.(19)

The tangential velocity is approximated with two straight lines, as

$$
\begin{array}{ll}
v_{x}=v_{s}(x)+a(x) y & {\left[y \leq y_{0}\right]} \\
v_{x}=0 & {\left[y \geq y_{0}\right]}
\end{array}
$$

Then

$$
\begin{array}{lr}
\Pi^{\prime \prime}(\eta)+\left(\alpha \eta+\beta \eta^{2}\right) \Pi^{\prime}(\eta)=0 & {\left[y \leq y_{0}\right]} \\
\Pi^{\prime \prime}(\eta)-\frac{\delta v_{y 0}}{D} \Pi^{\prime}(\eta)=0 & {\left[y \geq y_{0}\right]}
\end{array}
$$

where

$$
\begin{aligned}
& y_{0}=-v_{s} / a \\
& r v_{y 0}=-\left(r v_{s}\right)^{\prime} y_{0}-(r a)^{\prime} y_{0}^{2} / 2
\end{aligned}
$$

From Eqs.(12), (14) and (A-7), $\eta_{0}$ is derived as $\eta_{0} \equiv y_{0} / \delta=-1 /(B \sqrt{2 \alpha})$
Substituting Eq.(A-9) into Eq.(A-8), we obtain

$$
\frac{\delta v_{y 0}}{D}=-\frac{\eta_{0}}{4 r^{2} v_{s} D} \cdot \frac{d}{d x}\left(r^{2} v_{s}^{2} \delta^{2}\right)
$$

Combination of Eqs.(A-6), (A-9) and (A-10) yields

$$
\Pi^{\prime \prime}(\eta)+\frac{\alpha}{2} \eta_{0} \Pi^{\prime}(\eta)=0
$$

Integrating Eqs.(A-5) and (A-11) in each interval we obtain Eq.(19).

\section{Nomenclature}

$$
A=2 B / 3
$$

$a=\partial v_{x} /\left.\partial y\right|_{s}$

$B=a\left(D L / v_{s}^{3}\right)^{1 / 2}$

$D=$ binary diffusivity

$k=$ local mass transfer coefficient

$L=$ characteristic length defined by Eq.(13)

$r=$ distance from axis to interface

$v_{s}=$ interfacial tangential velocity

$v_{x}=$ tangential velocity component

$v_{y}=$ normal velocity component

$x=$ coordinate along interface

$y=$ coordinate normal to interface

$\langle$ Greek letters $\rangle$

$\alpha=$ constant

$\beta=$ constant

$\delta=$ a function defined by Eq.(12)

$\eta \equiv y / \delta$

$\eta_{0}=-1 /(B \sqrt{2 \alpha)}$

$\Pi=$ dimensionless normalized concentration, $\left(\omega_{A s}-\omega_{A}\right) /\left(\omega_{A s}-\omega_{A \infty}\right)$

$\tau \equiv \eta \sqrt{\alpha / 2}$

$\omega_{A}=$ mass fraction of solute

$\langle$ Suffix >

$s=$ interface

$\infty=$ bulk

\section{Literature Cited}

1) Beek, W. J. and C. A. P. Bakker: Appl. Sci. Res., A10, 241 (1961)

2) Lociel, A. C. and P. H. Calderbank: Chem. Eng. Sci., 19, 471 (1964)

3) Schlichting, H.: "Boundary-layer Theory", 6th ed., McGraw-Hill (1968)

4) Stewart, W. E., J. B. Angelo and E. N. Lightfoot: A.I.Ch.E. Journal, 16, 771 (1970) 